\title{
Einstein's contributions to atomic physics
}

\author{
Lorenzo J Curtis \\ Department of Physics and Astronomy, University of Toledo, Toledo, OH 43606, USA \\ E-mail: ljc@physics.utoledo.edu
}

Received 15 January 2009

Accepted for publication 16 January 2009

Published 29 April 2009

Online at stacks.iop.org/PhysScr/79/058101

\begin{abstract}
Many of the epoch-breaking papers that have been published by Einstein are remembered today as treatises dealing with various isolated phenomena rather than as direct consequences of a new unified world view. This paper traces the various ways in which ten papers published by Einstein during the period 1905-1925 influenced the development of the modern atomic paradigm, and illustrates how these discoveries can be made intuitive and pedagogically useful.
\end{abstract}

PACS numbers: $30.00,01.65 .+\mathrm{g}$

\section{Introduction}

Models for atomic structure are often associated with certain individuals, such as the 'atoms' of Thomson, Rutherford, Bohr, Schrödinger and Dirac. Although it is seldom specifically mentioned, many aspects of the development of these models were suggested or heavily influenced by Albert Einstein in papers that are primarily remembered for other applications.

Many of the epoch-breaking papers of Einstein are remembered today as anecdotal treatises dealing with isolated phenomena rather than for the new unified world view that they collectively contained. These papers are often classified by superficial tag lines such as 'Brownian motion', 'the photoelectric effect', 'special relativity', 'specific heats of solids', 'the Planck radiation law', 'general relativity', etc. However, rather than being just a series of separate solutions to diverse problems, these papers represented the development of a new perspective that changed the way we think about our physical universe.

The year 1905 was Einstein's 'annus mirabilis' — a date to set beside 1543 (when Nicolas Copernicus published De Revolutionibus Orbium Coelestium) and 1686 (when Isaac Newton published Philosophiae Naturalis Principia Mathematica). Between March and September of that year, Einstein produced four papers on three different subjects, any one of which could have assured his scientific immortality. A word of praise must be given to Paul Drude, the then editor of Annalen der Physik, who received and published with dispatch a battery of manuscripts from an obscure Swiss bureaucrat whose application to become a Privatdozent had been rejected.
It is clear why Drude accepted these manuscripts without hesitation. There is a striking clarity of exposition in these four papers that makes it apparent that these were not to Einstein a series of isolated studies of separate problems. The papers were instead a manifestation of a new enlightenment that had come into existence in the mind of Einstein that made these seemingly different problems become recognizable pieces of a puzzle that fell uniquely into place.

As an example of these interrelationships, the present paper will trace various ways in which ten papers [1-10] selected from Einstein's 1905-1925 work have influenced the development of the atomic hypothesis, or as Richard Feynman has called it 'the atomic fact', which he characterized as 'the most important discovery ever made'. The popular literature has an unfortunate tendency to portray Einstein's discoveries as counter-intuitive, implying that nothing is really quite as it seems. Another goal of this paper is to show that the opposite is true. Einstein has provided us with new perspectives from which to view the world. When we perceive nature with this enhanced vision it becomes clear that everything is exactly as it seems, and could not be otherwise. As Einstein said, 'the most incomprehensible thing about the world is that it is comprehensible'. This paper will attempt to illustrate ways in which Einstein's discoveries regarding atomic structure can be made intuitive, and simplify our understanding of the world around us.

\section{The existence of atoms}

Already in the fourth century BC, Democritus proposed that all matter consists of then inconceivably small particles, which he described as 'atoms', which meant 'indivisible'. 
The atomic picture was given added credence in 1662 when Robert Boyle discovered that air is compressible, and that this compression affects the pressure in an inverse relationship. From this, Boyle concluded that air must consist of discrete particles separated by a void. In 1808, John Dalton extended these studies to chemical measures of combining proportions, which indicated that atoms differ from each other in mass. Thus Dalton's work elevated atomism from a philosophical to a chemical theory. The discovery by Joseph-Louis Gay-Lussac that all gases expand to the same extent with a rise in temperature led Amedeo Avogadro to hypothesize in 1811 that equal volumes of all gases contain the same number of particles. Avagadro also indicated that these particles may be combinations of individual atoms, which he called 'molecules' (from the Latin 'moliculus', meaning 'small mass'). Unfortunately, despite the fame of Avogadro's name today, his suggestion received little attention at the time and was rejected by Dalton and ignored by Jöns Jakob Berzelius (the pre-eminent chemist of the day, whose symbolic system became the language of chemistry). Despite early philosophical formulations and the subsequent experimental development of atomism, the question remained whether atoms were real, or only a mnemonic device for coding chemical reactions. The acceptance by the scientific community of the existence of atoms did not occur until Einstein's May 1905 paper [2], the significance of which is usually concealed under the tag line 'Brownian motion'.

Despite the importance of the atomic nature of matter to the fields of chemistry and physics, Einstein's proof of their existence occurred as an outgrowth of studies by the Scottish botanist Robert Brown. In 1827, Brown was viewing under a microscope a suspension of pollen grains in water and noticed that the individual grains were moving about irregularly. He initially thought that this might be a result of animate life hidden within the grains, but found that the same erratic motion occurred when the pollen grains were replaced with ground glass or dye particles. Although he had no explanation for the phenomenon, he reported the result, which has since been called 'Brownian motion'.

Brownian motion found an explanation in the kinetic theory of gases developed in about 1860 by the Scottish mathematician James Clerk Maxwell. Amazingly, the kinetic theory itself owes its initial formulation not to physics and chemistry, but to the social sciences [11]. After the French revolution, the great mathematician Pierre-Simon Laplace was required to adapt his work to serve the revolutionary goals, and to educate the populace through a series of public lectures. To this purpose, Laplace adapted his studies of probability theory (initiated to rescue 'Laplacian determinism' from the measurement imprecision that he attributed to 'human weakness' rather than to innate indeterminacy) to demography and actuarial determination. Laplace's lectures were attended by the Belgian astronomer Adolphe Quetelet, who was inspired by them to formulate the study of 'Staatswissenschaft', the forerunner of the modern statistical social sciences. Quetelet's work was heralded as a cure for societal ills, and was championed by the social reformer Florence Nightingale. This subsequently inspired James Clerk Maxwell, through his reading of an essay on Quetelet's work written by John Herschel (the astronomer), to adopt a strategy using Laplace's probabilistic methods as a basis for his kinetic theory of gases. Maxwell's formulation of statistical mechanics marked a turning point in physics, since (in contrast to Laplacian determinism) it presupposed the operation of chance in nature. Thus, in this case, the 'exact sciences' borrowed from the 'social sciences'.

It should be noted that the contributions of Florence Nightingale were of great significance. Although usually remembered as a pioneer in nursing, she was also one of the leading mathematicians of her time. She developed new techniques of analysis and innovations in the collection, tabulation, interpretation and graphical display of statistical data. During the Crimean War, she invented the now familiar 'polar-area diagram' (pie-chart) to dramatize the needless deaths caused by unsanitary conditions in military hospitals. Although she lived to see the Einstein era (she died in 1910), her mathematical interest can be traced to the post-revolution lectures of Laplace (she was six years old when Laplace died in 1827).

Although the kinetic theory of matter provided a qualitative explanation of Brownian motion, a quantitative formulation was still lacking. This was provided by Einstein in his 1905 doctoral dissertation and in the May paper of his annus mirabilis. Einstein attacked this problem using the same probabilistic methods developed by Laplace, here the 'Random Walk', which is a sequence of discrete steps, each in a random direction. For a large object, the number of molecules striking on all sides and from all angles is approximately equal, so there is no overall effect. For a smaller object, the number of molecules striking it during a short interval can be reduced by statistical fluctuations, and small differences in bombardment can buffet the object about to an observable degree. The larger the size and mass of the molecules, the larger the size of the object for which this difference in bombardment can produce detectable results. Using the methods of probability, Einstein was able to compute the distribution of distances by which the pollen grains would be expected to migrate as a function of the size of the molecules. Theodor Svedberg at the University of Uppsala had also suggested a molecular explanation for Brownian motion, but it was Einstein who produced the mathematical formulation that demonstrated its correctness.

Publication of the Brownian motion paper quickly led to the determination of Avogadro's number. Before Einstein, the number of molecules in a gram molecular weight was assumed to be constant, but had no definite quantity. By 1908, Jean Perrin used Einstein's paper to make the first estimate of the number of molecules in a mole of any substance. Within a decade, $6.02 \times 10^{23}$ atoms per gram-mole was on its way to becoming one of the most widely known of the fundamental constants.

In the period prior to 1905 , a leading proponent of the atomic hypothesis was Ludwig Boltzmann. Boltzmann extended Maxwell's work in statistical mechanics to obtain the Maxwell-Boltzmann distribution, which connected atoms with macroscopic phenomena. Boltzmann's atomistic ideas were bitterly attacked by scientists such as Wilhelm Ostwald, and this has been cited as a possible contributing cause of the depression and mental breakdown that led to Boltzmann's suicide in 1906. If so, it is ironic that the means that would 
ultimately vindicate his work were already available at the time of his death.

Eugene Wigner has recounted that a book from which he studied that was written before 1905 stated that 'atoms and molecules may exist, but this is irrelevant from the point of view of physics'. After Einstein's analysis, it was not only known that atoms exist, but anyone with a ruler and a stop watch could measure their size [12]. While this paper never captured the popular imagination, in many ways it is the one that had the most profound effect on contemporary thought.

\section{The discrete nature of the photon}

Of the annus mirabilis papers, only the one of March 1905 [1] was considered by Einstein himself to be 'revolutionary'. This paper is given the tag line 'photoelectric effect', although that subject plays only a minor role. The primary impact of the paper was to unequivocally establish the integrity of the photon as a localizable particle possessing a discrete amount of energy. Einstein began with the observation that the entropy of a system (the ratio of energy to temperature) varied with the volume of a closed cavity for light in the same way as it did for an ideal gas. Since the gas entropy relationship was deduced from the assumption that the gas existed as discrete molecules, Einstein reasoned that light was also emitted in discrete entities. The paper then went on to apply this revolutionary conclusion to the Stokes rule of photoluminescence, to the photoelectric effect and to the ionization of gases by ultraviolet light. The historical emphasis given to the photoelectric effect application rather than to the new physics that Einstein proposed is therefore misleading. It was for this discovery that Einstein was awarded the Nobel Prize in Physics in 1921.

In placing this discovery by Einstein in the context of our understanding of atomic structure, it is important to clarify historical shifts in the meaning of certain words. The standard textbook presentation of this discovery by Einstein is clouded by multiple meanings associated with the word 'quantum' as used in different contexts. It is often incorrectly asserted that light quanta had been proposed in 1900 by Max Planck in his formulation of blackbody emission. Planck's formulation did not contain any suggestion concerning the particle nature of light, since he instead associated the denumerable discreteness to fictitious oscillators that he assumed produced the light. Had Planck suspected that he was counting discrete light particles rather than discrete resonators, it would have been more reasonable to denote them as 'corpuscles' instead of 'quanta'. In $1899 \mathrm{~J} J$ Thomson had used the word 'corpuscle' (the diminutive of the Latin word 'corpus', or body) to describe his discovery that the electron is a discrete particle. Newton had used 'corpuscule' for light particles prior to the development by Thomas Young of the wave model. In contrast, Planck assumed that discrete resonators produced quanta of energy, but the electromagnetic waves so produced were considered to be continuously distributed over space.

Following Planck's nomenclature, Einstein used the words 'Raumpunkten lokalisierten Energiequanten' to describe what we would now call photons. The name 'photon' was first suggested in 1926 by the American chemist Gilbert N Lewis [13], as a way to differentiate between discrete light particles and the quantum numbers that prescribe the discrete energy levels of a bound system, which were introduced in 1913 by Niels Bohr. It is interesting to note that the Planck-Einstein use of the word 'quantum' corresponds to the modern concept of 'second quantization' (the quantization of the field in quantum electrodynamics), whereas the Bohr-Sommerfeld quantization is now called 'first quantization' (the discrete units of action characteristic of a bound state of an atom). Thus, these two important concepts are introduced using a historical framework in which 'second quantization' was postulated before 'first quantization'.

The continuing discussions in modern physics textbooks of the fictitious Planckian oscillators and the use of the word quanta to denote photons are unfortunate. Clearly, blackbody radiation involves continuum collisions of free electrons, and should not be confused with transitions between the bound states of a quantum mechanical harmonic oscillator. Similarly, the use of the word quantum to denote a photon confuses its usage as the unit of action that quantizes a stationary state.

One cannot leave the subject of the photoelectric effect without mentioning the relationship between Einstein and Phillipp Lenard, upon whose data the application to the photoelectric effect at the end of the paper was based. Lenard and Johannes Stark were probably the two most vehement Nazi supporters among German scientists, and their savage anti-Semitic attacks on Einstein's theories were a factor causing him to leave Germany. In an interview, Robert Shankland [14] discussed Lenard with Einstein. Shankland cited as a true measure of Einstein's objectivity the fact that he referred to Lenard's work 'with complete fairness and not the slightest trace of malice or bitterness'.

\section{The Einstein-Brillouin-Keller (EBK) quantization}

As mentioned earlier, the word 'quantum' is still often used to describe the fact that photons are localized particles, each possessing quantifiable values for energy and momentum. This is not to be confused with the word 'quantization', which refers to the fact that a condition for observation of a bound system requires that its 'action' fulfills certain integer relationships with Planck's constant. The action integral is of the form

$$
\text { Action } \equiv \oint p_{i}\left(q_{i}\right) \mathrm{d} q_{i} .
$$

While it is sometimes stated that the energy levels of a bound system are quantized, it is the action that is quantized in units of Planck's constant, and the discrete energy levels occur only as a secondary consequence of action quantization.

The postulation of quantized action was made in 1913 by Niels Bohr, in association with the theoretical specification of the discrete line spectrum of the hydrogen atom. In the three-dimensional formulation of a central potential there are three action integrals corresponding to the spherical polar coordinates $r, \theta$ and $\phi$

$$
\oint p_{r}(r) \mathrm{d} r ; \quad \oint p_{\theta}(\theta) \mathrm{d} \theta ; \quad \oint p_{\phi}(\phi) \mathrm{d} \phi .
$$


Bohr assumed the unrealizably special case of an exactly circular orbit, which allowed him to limit consideration to the azimuthal action integral. Assuming this to be an integer multiple of Planck's constant

$$
n_{\phi} \hbar=\frac{1}{2 \pi} \oint p_{\phi}(\phi) \mathrm{d} \phi,
$$

he obtained the familiar expression for the Balmer energy.

Arnold Sommerfeld and William Wilson later extended this treatment relativistically to include elliptic orbits, assuming the quantizations

$$
n_{\theta} \hbar=\frac{1}{2 \pi} \oint p_{\theta}(\theta) \mathrm{d} \theta ; \quad n_{r} \hbar=\frac{1}{2 \pi} \oint p_{r}(r) \mathrm{d} r .
$$

This formulation is known as the Bohr-Sommerfeld-Wilson (BSW) quantization. It quickly leads to results that conflict with experiment, and it is routinely dismissed in most modern physics textbooks. However, the origin of these errors is not in the general approach, but in a simple mathematical mistake made in its application. If correctly formulated, this action quantization can be very useful and provides many valuable results.

While the azimuthal quantization made by Bohr is proper, the zenith and radial quantizations are incorrect, as was shown by Einstein in 1917 [9]. In this paper, Einstein pointed out that the contour integral for the $r$ and $\theta$ coordinates do not involve rotations, but rather librations, which oscillate between two turning points (caustics). In such cases the integral involves a contour over two Riemannian sheets, which leads to a phase jump at each turning point. The problem was subsequently studied by Léon Brillouin, Joseph B Keller and Viktor Pavlovich Maslov, and the quantization can be correctly stated as [16]

$$
\left(n_{i}+\left(\mu_{i} / 4\right)\right) \hbar=\frac{1}{2 \pi} \oint p_{i}\left(q_{i}\right) \mathrm{d} q_{i} .
$$

Here, $\mu$ is the Maslov index, which corresponds to the number of turning points. Thus $\mu=0$ for rotations, $\mu=1$ for motions with a single turning point (such as field emission from a cold cathode or the tip of a scanning tunneling microscope), $\mu=2$ for librations between two turning points and $\mu=4$ for an infinite square well (which has two turning points and two reflections).

This formalism is known as the EBK quantization. It yields correct values for most of the standard problems of quantum mechanics, and has advantages over fully quantum mechanical treatments in a number of applications. The procedure is not only applicable to atomic physics, but is also the basis for the computation of the RKR (for Ragnar Rydberg, Oskar Klein and Albert Lloyd George Rees) method for computing the Franck-Condon factors in molecular physics.

For spherically symmetric potentials, the angular action integrals can be performed. In terms of the modern quantum mechanical notation $m_{\ell} \equiv \pm n_{\phi}$ and $\ell \equiv n_{\theta}+n_{\phi}$, these quantizations yield, for the orbital angular momentum $L$ and its $z$-projection $L_{z}$,

$$
L=(\ell+(1 / 2)) \hbar ; \quad L_{z}=m_{\ell} \hbar .
$$

This is in agreement with the modern quantum mechanical result for $L_{z}$, and $L^{2}=\left(\ell^{2}+\ell+(1 / 4)\right) \hbar^{2}$ approaches the quantum mechanical result $\ell(\ell+1) \hbar^{2}$ in the correspondence limit. For the Coulomb and isotropic harmonic oscillator potentials, the EBK radial quantization yields the same energy levels as modern quantum mechanical calculations.

In this little-known paper [9, 17], Einstein corrected an error in the formulation of the BSW quantization, generalized the method to problems with several degrees of freedom that are not separable and also addressed a profound question regarding classical and quantum chaos. In 1887 the King of Sweden had sponsored as a contest a challenge to show rigorously that the solar system is stable. The French mathematician, astronomer and physicist Henri Poincaré submitted an entry, which at first appeared to have succeeded, but Poincaré subsequently discovered an error. Poincaré's correction of that error is generally regarded as the birth of chaos theory. In Einstein's 1917 paper, he pointed out that the method (later called EBK) fails if there do not exist a number of integrals of motion equal to the number of degrees of freedom, that is, unless the system is integrable. He suggested that the non-integrable case is typical of classical dynamics, but indicated that the quantum situation was an open question. While this aspect of the paper was ignored until the 1960s, the problem noted by Einstein is fundamental and has never fully been overcome.

It is unfortunate that this very important and conceptually fertile paper by Einstein has been almost completely overlooked by textbook writers (although not by researchers).

\section{Relativity and fine structure}

The titular subject of the June paper of Einstein's annus mirabilis is seldom mentioned in discussions of its implications. The title of the paper is 'On the electrodynamics of moving bodies' [3] and its first sentence states 'It is known that Maxwell's electrodynamics - as usually understood at the present time-when applied to moving bodies, leads to asymmetries which do not appear to be inherent in the phenomena'. While this paper is usually referred to by the tag line 'special relativity', its intent was not to describe rocket ships traveling at velocities near the speed of light, but rather to describe magnetic fields produced by currents corresponding to electron drift speeds of tens of millimeters per second or less [15]. Thus the results of this paper should appear in every elementary physics textbook between the chapters on Coulomb's law and the Biot-Savart law, but instead it is deferred to the end of the book or saved for a subsequent advanced course for physics majors.

The beauty and simplicity of the relativistic formulation can be seen from the following pedagogic example [16]. Consider a copper wire $1 \mathrm{~mm}$ in diameter through which a current of $1 \mathrm{~A}$ passes. Assuming one conduction electron per copper atom, this current corresponds to a drift speed of $\frac{1}{10} \mathrm{~mm} \mathrm{~s}^{-1}$.

One of the results of special relativity is the fact that if two extended objects move relative to each other, each underestimates the length of the other along the direction of motion. Thus, to an observer who is stationary relative to the wire, the negative electron charge will appear slightly denser. 


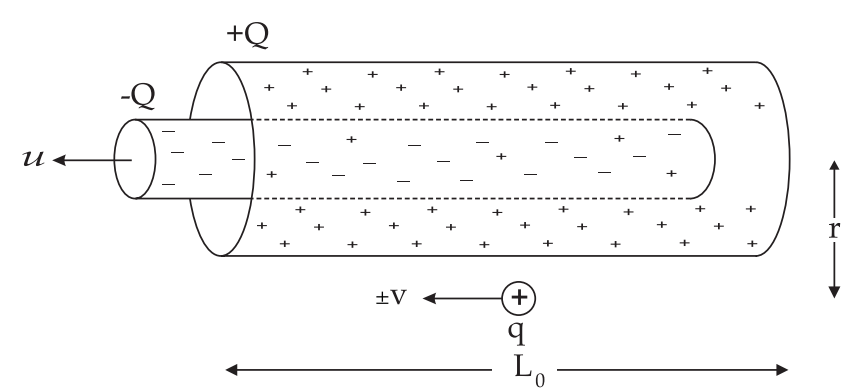

Figure 1. Model for a current.

This is a very small effect, but it is greatly enhanced as seen by a charge that moves with a significantly larger speed relative to the wire. A model for such a current is shown in figure 1 .

If a segment of the wire of length $L_{0}$ contains a positive charge $Q$ and a free-electron charge $-Q$ with a drift speed $u$, and the test charge $q$ has a speed $\pm V$ (either parallel or antiparallel to the electron drift) at a distance $r$ from the center of the wire, the Coulomb force on the test charge is given by

$$
\begin{aligned}
F= & \frac{2 K q}{r}\left[\frac{Q}{L_{0} \sqrt{1-\left(V^{2} / c^{2}\right)}}\right. \\
& \left.-\frac{Q}{L_{0} \sqrt{1-\left(( \pm V-u)^{2} / c^{2}\right)}}\right] .
\end{aligned}
$$

Here, we have included the apparent relativistic contraction of the two line charges as seen by the moving test charge. This can be binomial expanded to yield

$$
\begin{aligned}
F \approx & \frac{2 K q}{r} \frac{Q}{L_{0}}\left[\left(1+\frac{V^{2}}{2 c^{2}}+\cdots\right)\right. \\
& \left.-\left(1+\frac{V^{2} \mp 2 V u+u^{2}}{2 c^{2}}+\cdots\right)\right] .
\end{aligned}
$$

To first order in $u / c$ this becomes

$$
F \approx \pm \frac{2 K q V}{r c^{2}}\left(-\frac{Q u}{L_{0}}\right)
$$

Although $u$ is small, $Q / L_{0}$ is large, and their product can be identified as the current in the wire. Thus we can define

$$
I \equiv-Q u / L_{0} ; \quad B \equiv 2\left(K / c^{2}\right) I / r ; \quad F= \pm q V B,
$$

where the plus sign denotes repulsion if the test charge moves in the same direction as the electron drift, and the minus sign denotes attraction if the test charge moves opposite to the electron drift. This simple model demonstrates that the Biot-Savart law is a consequence of Coulomb's law and relativity, and not a separate experimental fact.

While this paper is probably the one considered the most radical by the general public, the basic concepts of relativity had been in the scientific air for a very long time. Already in 1887, Woldemar Voigt [18] had studied transformations of the electromagnetic wave equation between space-time coordinate systems for which the wave speed is invariant, and obtained the equations that are today called the Lorentz transformations. Thus Voigt conceived the idea of a universal value for the speed of light and demonstrated that the Doppler shift in frequency is incompatible with Newtonian absolute time. In 1895 the transformations were rederived in another context by George FitzGerald and Hendrik Antoon Lorentz. Although the Lorentz and Voigt corresponded frequently, the 1887 paper by Voigt had escaped the attention of Lorentz, who belatedly cited the work of Voigt in the 1908 edition of his book The Theory of Electrons. Prior to Einstein's presentation, Henri Poincaré presented a lecture on the 'Principle of Relativity' at the 1904 St. Louis World's Fair (the scene depicted in the 1940s Hollywood musical 'Meet $\mathrm{Me}$ in St. Louis'). However, the clarity, thoroughness and transparency of Einstein's presentation brought all of the various aspects of relativity together in a manner that certainly justifies his priority.

There are many features of relativity that influenced the development of atomic theory. One involves the relativistic kinetic energy $T$, which can be written in terms of the mass $m$ and momentum $p$ as

$$
\begin{aligned}
T & =\sqrt{\left(m c^{2}\right)^{2}+(p c)^{2}}-m c^{2} \\
& \cong m c^{2}\left[1+\frac{1}{2}\left(\frac{p}{m c}\right)^{2}-\frac{1}{8}\left(\frac{p}{m c}\right)^{4}+\ldots\right]-m c^{2} .
\end{aligned}
$$

In terms of the nonrelativistic kinetic energy $T_{0} \equiv p^{2} / 2 m$, this becomes

$$
T \cong T_{0}-\left(T_{0}^{2} / 2 m c^{2}\right)+\cdots,
$$

and the correction $\Delta E$ to the nonrelativistic energy is

$$
\langle\Delta E\rangle=\langle T\rangle-\left\langle T_{0}\right\rangle=\left\langle T_{0}^{2}\right\rangle / 2 m c^{2} .
$$

(The angular brackets indicate orbital averages.) For a Coulomb potential, the total nonrelativistic energy $E_{0}=T_{0}-$ $(\kappa / r)$ (here and henceforth $\left.\kappa \equiv e^{2} / 4 \pi \epsilon_{0}\right)$, so

$$
\left\langle T_{0}^{2}\right\rangle=\left\langle\left(E_{0}+(\kappa / r)\right)^{2}\right\rangle=E_{0}^{2}+2 E_{0}\langle\kappa / r\rangle+\left\langle\kappa^{2} / r^{2}\right\rangle
$$

and the virial theorem yields $E_{0}=-\langle\kappa / r\rangle / 2$; hence

$$
\langle\Delta E\rangle=-\frac{\kappa^{2}}{2 m c^{2}}\left[\left\langle r^{-2}\right\rangle-\frac{3}{4}\left\langle r^{-1}\right\rangle^{2}\right] .
$$

In terms of the semimajor axis $a$ and semiminor axis $b$ of the elliptic orbit, this is

$$
\langle\Delta E\rangle=-\frac{\kappa^{2}}{2 m c^{2}}\left[\frac{1}{a b}-\frac{3}{4 a^{2}}\right],
$$

where $a_{0}$ is the Bohr radius. This is the expression for the energy correction to the advance of the perihelion of the planet Mercury as predicted by Einstein on the basis of special relativity ( 7.2 arcseconds century $^{-1}$ ). The EBK quantization for the hydrogen atom yields the values

$$
a=a_{0} n^{2} ; \quad b=a_{0} n(\ell+(1 / 2)) .
$$

Inserting these values yields the correct quantum mechanical expression for the relativistic momentum fine structure correction

$$
\langle\Delta E\rangle=-\frac{R \alpha^{2}}{n^{3}}\left[\frac{1}{\left(\ell+\frac{1}{2}\right)}-\frac{3}{4 n}\right],
$$

where $R=\kappa / 2 a_{0}$ and $\alpha=\sqrt{\kappa / m c^{2} a_{0}}$ are the Rydberg and fine structure constants. 
In most modern physics textbooks, the treatment of Einstein's calculation of the advance of the perihelion of Mercury and the calculation of the fine structure of the hydrogen atom are treated in very different ways in widely separated chapters. Combining the two in this manner can be both conceptually and calculationally effective.

In addition to this fine structure correction for the relativistic momentum of the electron, there is also a second relativistic correction caused by magnetic interactions. This is due to the interaction between the intrinsic magnetic moment of the electron and the magnetic field set up by the relative motion of the nucleus as seen by the orbiting electron. The magnetic field can be written as

$$
\mathbf{B}=\frac{K}{c^{2}} \frac{e(\mathbf{r} \times \mathbf{v})}{r^{3}} .
$$

This circulation of charge $e(\mathbf{r} \times \mathbf{v})$ can be written in terms of the circulation of mass, which is the orbital angular momentum

$$
\mathbf{L}=m(\mathbf{r} \times \mathbf{v}) .
$$

The anomalous magnetic moment of the electron is

$$
\mu_{\mathrm{s}}=-g_{\mathrm{e}} \frac{e}{2 m} \mathbf{S}
$$

where $g_{\mathrm{e}} \approx 2$ is the $g$-factor of the electron. These equations can be used to compute the interaction energy $\Delta E=-\left\langle\mu_{\mathrm{s}} \cdot \mathbf{B}\right\rangle$. However, it is necessary to transform the result into the normal frame in which the nucleus is at rest, and this involves an additional relativistic correction called the 'Thomas precession'. This causes the electron $g$-factor to be replaced by $g_{\mathrm{e}} \rightarrow g_{\mathrm{e}}-1 \approx 1$, and yields the result

$$
\Delta E=\frac{\kappa}{2(m c)^{2}}\left\langle\frac{\mathbf{L} \cdot \mathbf{S}}{r^{3}}\right\rangle=\frac{\kappa}{2(m c)^{2}} \frac{\langle\mathbf{L} \cdot \mathbf{S}\rangle}{b^{3}},
$$

where $b$ is the semiminor axis of the ellipse.

This correction also has a connection to Einstein's general relativistic formulation of the advance of the perihelion of the planet Mercury. When general relativity is applied to the gravitational problem, the Schwarzschild solution of the Einstein field equations assumes the form [19]

$$
\langle\Delta E\rangle=\frac{\kappa L^{2}}{(m c)^{2}}\left\langle r^{-3}\right\rangle .
$$

So, just as in the case of the atomic spin-orbit interaction, there is here another correction beyond the relativistic kinetic energy of special relativity that depends on the inverse cube of the radius vector. In the case of the advance of the perihelion of Mercury, use of the general relativity calculation corrects the value obtained from special relativity by a factor of six, which exactly matches the observed result 43 arcseconds century ${ }^{-1}$. Einstein first made this calculation in November 1915 and in the following January he is said to have written to Paul Ehrenfest saying, 'For a few days I was beside myself with joyous excitement'.

Using the EBK quantization for the value of $b$, the magnetic contribution to the fine structure of hydrogen is

$$
\langle\Delta E\rangle=R \alpha^{2} \frac{\langle\mathbf{L} \cdot \mathbf{S}\rangle}{n^{3}(\ell+(1 / 2))^{3}} .
$$

The corresponding expression obtained by applying perturbation theory to the Schrödinger equation is

$$
\langle\Delta E\rangle=R \alpha^{2} \frac{\langle\mathbf{L} \cdot \mathbf{S}\rangle}{n^{3} \ell(\ell+(1 / 2))(\ell+1)},
$$

which illustrates another advantage of the EBK quantization. The perturbation solution obtained using the nonrelativistic Schrödinger wave functions is indeterminant for s-states $(\ell=0)$, since these wave functions sample $r=0$ where the Coulomb potential diverges. This artifact of the nonrelativistic calculation requires an additional correction called the Darwin term. This is not a problem in the EBK formulation because, owing to the Maslov index, an s-state has a small but finite perihelion.

The origin of this problem extends to the Dirac equation and lies in choices in evaluating the nonrelativistic limit. Different operators occur in the Dirac Hamiltonian (which includes both the positive and negative energy states) and the nonrelativistic limit of the Pauli Hamiltonian (with its position and spin operators). The proper transformation of the Dirac Hamiltonian to the Pauli Hamiltonian in the presence of an electromagnetic field was obtained in 1950 by Leslie L Foldy and Siegfried A Wouthuysen [20]. In this transformation, a point particle that moves smoothly in the Dirac space-time coordinates acquires a jittery motion (or 'Zitterbewegung') in the Pauli representation. Thus it dances about in a region of the order of its Compton wavelength under the influence of its absorption and re-emission of virtual photons from the electromagnetic field. Because of this effect the behavior of a point electron exhibits some properties characteristic of a particle of finite extension, which explains the angular momentum and magnetic moment exhibited by the electron.

One of the important aspects of the impact of relativity on atomic physics involves symmetry under time reversal. It is interesting to note that the development of the cinema was proceeding at the same time as the development of relativity. In Paris in 1895, Auguste and Louis Lumière made the first public screening of a moving picture. Their Cinematograph not only produced realistic moving images, but during the rewind process it provided the first visualization of time-reversed motion. It is therefore not surprising that the general public was intrigued by the fundamental significance that relativity gives to time reversal.

It is interesting to note that, despite Einstein's formulation of special relativity in 1905, in 1925 the major advance in atomic theory involved the nonrelativistic Schrödinger equation. Dirac has recounted in [21] a story told to him by Schrödinger regarding his development of the wave equation. In trying to generalize the ideas of DeBroglie regarding waves associated with particles, Schrödinger considered a mathematical operator constructed from the relativistic energy relationships governing the Coulomb potential. He began with what Dirac called 'Schrödinger's first wave equation'

$$
\left(E+m c^{2}+(\kappa / r)\right)^{2} \psi=\left[\left(m c^{2}\right)^{2}+(p c)^{2}\right] \psi .
$$

Schrödinger applied this equation to the behavior of the electron in the hydrogen atom, but obtained results that disagreed with experiment. This disappointment caused him to abandon the work for several months. However, he 
later returned to this study and rewrote the equation in an approximate way, neglecting the refinements required by relativity. By taking the square root of the operators on both sides of the equation above, and expanding the right side in powers of $(p / m c)^{2}$, he obtained what Dirac called 'Schrödinger's second wave equation'

$$
(E+(\kappa / r)) \psi=\left(p^{2} / 2 m\right) \psi .
$$

To his surprise, Schrödinger found that the results obtained using this rough approximation were in agreement with available observations.

Both equations fail to account for the intrinsic spin of the electron, but they omit this crucial element in different ways. 'Schrödinger's first equation' is now known as the Klein-Gordon equation, and describes a spinless particle. 'Schrödinger's second equation' is the normal Schrödinger equation, and represents a factorization of the spatial and spin portions of the wave function. The fully relativistic treatment is given by the Dirac equation, in which Schrödinger's first equation is written as a complex factorization rather than a square root

$$
\left(E+m c^{2}+(\kappa / r)\right) \psi=\left(\mathbf{p c}+\mathrm{i} m c^{2}\right) \psi .
$$

The solution to this equation is not a scalar wave function but rather a four-component vector. Like the Foldy-Wouthuysen transformation, Schrödinger's first and second equations represent different nonrelativistic limits of the relativistic equation: the first retains Lorentz covariance but loses electron spin; the second forfeits Lorentz covariance but retains the possibility of including electron spin as a multiplicative Pauli correction.

The fact that the relativistic Dirac equation produced four solutions led to many new discoveries. The solutions could be classified as the two spin states of the electron, and a time-reversed counterpart that was ultimately identified as the positron, the first known antiparticle. The electron and the positron were subsequently connected to the photon in a most useful way through the development of quantum electrodynamics.

In his acceptance speech upon being awarded the Nobel Prize [22], Richard Feynman recalled a picture suggested by John Wheeler, in which there is only one single electron in the universe, which doubles back and forth between the past and future by moving forward and backward in time. This is based on the fact that when two energetic photons collide, an electron-positron pair creation occurs, and when an electron-positron collision occurs they annihilate to form two energetic photons. Feynman and Wheeler pictorially represented these processes as an electron encountering an energetic photon, whereupon both reverse their directions in time (a photon is its own antiparticle). The electron (in its positron disguise) then proceeds backward in time until it encounters another energetic photon, whereupon both reverse their time directions again. This process continues ad infinitum, and each transit through the 'here-now' is interpreted as an electron or positron. A diagram of this process is given in figure 2

While this picture ignores the apparent disparity between the numbers of electrons and positrons in the known universe,

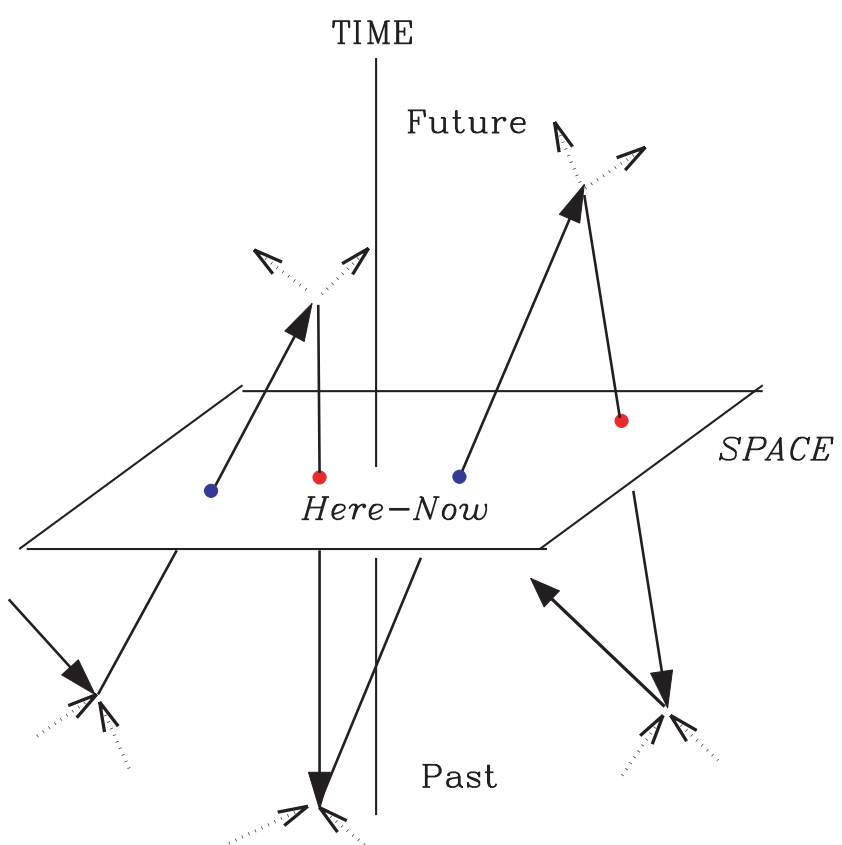

Figure 2. The universal electron.

it illustrates an essential feature of quantum electrodynamics: all of the electrons in the universe are coupled to the electromagnetic field of virtual photons, and thereby all are coupled to each other. Thus, rather than considering the electron and photon as two different particles, it becomes useful to consider the 'dressed electron', which, by virtue of its charge, is inseparable from its virtual photon field.

\section{Emission and absorption}

Einstein's 1917 paper on 'The quantum theory of radiation' [8] was the first formulation of a number of different physical processes. It was the first definition of the Einstein $A$ and $B$ coefficients that govern spontaneous emission, stimulated emission and absorption. It was the first consideration of stimulated emission, which provided the basis for the maser and laser long before their development. It was the first demonstration that the Planck continuum radiation law can be deduced from the Bohr theory of the atom and the Boltzmann statistical law for an ensemble in thermal equilibrium.

However, a major portion of the paper was devoted to a discussion of the fact that photons must obey not only conservation of energy, but also conservation of momentum. Einstein reasoned that, while the emission of radiation can be considered as consisting of spherical waves, the absorption of radiation is a 'fully directed event' involving a plane wave. Thus, while emission could be treated through conservation of energy, Einstein showed that absorption required the consideration of the directed momentum of the photon and the recoil of the absorbing atom. Although physics textbooks cite the 1923 measurement by Arthur Holly Compton as the experimental proof that photons conserve momentum, Einstein had already confirmed this using blackbody radiation in 1917.

Einstein's introduction of the $A$ and $B$ coefficients was conceptual, assuming (in analogy to nuclear decay processes) 


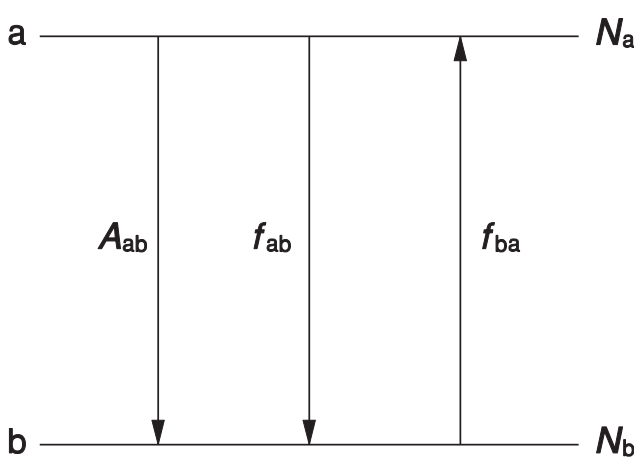

Figure 3. Emission and absorption.

that the various rates were proportional to the instantaneous number of atoms multiplied by a constant coefficient. Since this was done before the relationships of these coefficients to the electric dipole matrix element were formulated, Einstein had to deduce the ratio of $A$ to $B$ empirically, by forcing it to conform to the known quantities that occur in the Planck Law. Since these relationships are now known, it is possible to generalize Einstein's formulation, obtaining the Planck Law from first principles without recourse to the empirical specification of any factors.

Einstein's $A$ coefficient is still in use, and is the standard spontaneous transition probability rate $A_{i j}$. The use of the $B$ coefficient has largely been replaced by the oscillator strength $f_{j i}$, which is a dimensionless quantity that relates the absorption of radiation by a classical simple harmonic oscillator to that of the corresponding quantum mechanical system. The Einstein $B$ coefficient differs from the $A$ coefficient in that it involves the energy/time rather than photons/time.

A diagram indicating two radiation-coupled levels is shown in figure 3. In terms of the $A$ and $f$ quantities, the population equation for an arbitrary pair of levels $a$ and $b$ with populations $N_{a}$ and $N_{b}$ (where $E_{a}>E_{b}$ ) in the presence of a photon flux density $\rho(\omega)$ is

$-\frac{\mathrm{d} N_{a}}{\mathrm{~d} t}=\frac{\mathrm{d} N_{b}}{\mathrm{~d} t}=N_{a} A_{a b}+\frac{\pi \kappa}{m}\left[N_{a} f_{a b}+N_{b} f_{b a}\right] \rho(\omega)$.

where the three contributions on the right side are spontaneous emission, stimulated emission and absorption. At equilibrium $-\mathrm{d} N_{a} / \mathrm{d} t=\mathrm{d} N_{b} / \mathrm{d} t=0$, and this equation can be solved for $\rho(\omega)$

$$
\rho(\omega)=-\frac{m N_{a} A_{a b}}{\pi \kappa\left(N_{b} f_{b a}+N_{a} f_{a b}\right)} .
$$

The populations can be eliminated by the use of the Boltzmann distribution

$$
\frac{N_{b}}{N_{a}}=\frac{g_{b}}{g_{a}} \exp \left[-\left(E_{b}-E_{a}\right) / k T\right]=\frac{g_{b}}{g_{a}} \mathrm{e}^{-\hbar \omega / k T} .
$$

The relationship between absorption and stimulated emission oscillator strengths involves a sign and the degeneracies $g_{b} f_{b a}=-g_{a} f_{a b}$. This yields

$$
\rho(\omega)=\frac{m}{\pi \kappa} \frac{g_{a} A_{a b}}{g_{b} f_{b a}} \frac{1}{\left(\mathrm{e}^{\hbar \omega / k T}-1\right)} .
$$

The relationship between emission and absorption is

$$
g_{a} A_{a b}=\frac{2 \kappa \omega^{2}}{m c^{3}} g_{b} f_{b a}
$$

from which the distribution in photon space is deduced

$$
\rho(\omega)=\frac{2 \omega^{2}}{\pi c^{3}} \frac{1}{\left(\mathrm{e}^{\hbar \omega / k T}-1\right)} .
$$

To connect this to the Planck distribution law, which was formulated as an energy density rather than a photon density, we multiply by $\hbar \omega$

$$
\hbar \omega \rho(\omega)=\frac{2 \hbar \omega^{3}}{\pi c^{3}} \frac{1}{\left(\mathrm{e}^{\hbar \omega / k T}-1\right)} .
$$

It should be emphasized that, while this description treats two levels $a$ and $b$, there is no requirement that specifies whether these are bound or continuum levels. While Einstein used this formalism to describe the Planck law for continuum radiation emitted by free electrons in a plasma or in a metal, it is still used today to describe transitions between stationary states of bound systems. In 1924, Bose and Einstein developed a more direct method of deducing the Planck distribution using quantum statistics, but this 1917 formulation still remains the method used for describing bound state emission and absorption.

It is interesting that Einstein formulated the concept of spontaneous emission not as a radiation rate but as a rate of change of probability between stationary states. The fact that Einstein here embraced a probabilistic formulation indicates that there was a greater degree of subtlety than is normally accorded to his later objections (Gott würfelt nicht!) to this aspect of quantum mechanics and the Copenhagen interpretation.

\section{Quantum statistics}

Although the 1917 paper [8] by Einstein had developed new concepts such as spontaneous and stimulated emission, absorption, and conservation of vector momentum by photons to bear on the derivation of the Planck radiation law, a new and much simpler method for obtaining this result was revealed to him in 1924.

In that year, Einstein received a manuscript in English from a young Indian physicist, Satyendra Nath Bose [23], which set forth a theory in which radiation was treated as a photon gas. Such approaches had been tried before using standard Maxwell-Boltzmann statistics, but they yielded the Wien rather than the Planck distribution. However, by changing the statistical method by which he counted the states of the gas to describe indistinguishable particles, Bose had obtained a new distribution function that produced the correct Planck distribution.

Daniel Kleppner [24] has imagined that this could have been a 'forehead slapping moment' in which Einstein might have exclaimed 'Why didn't I think of that?' Einstein translated Bose's paper into German and immediately forwarded it to Zeitschrift für Physik with a recommendation for publication. Einstein then set about to apply Bose's 
distribution (now known as the Bose-Einstein statistics) to a gas of material particles.

While Einstein was working on this project, he received another manuscript, this one a doctoral dissertation written in Paris by Louis de Broglie. In two papers published in 1909 [5, 6], Einstein had shown that statistical fluctuations in thermal radiation fields display both particlelike and wavelike behavior, which was the first indication of what came to be called complementarity. The thesis of de Broglie proposed the concept of 'pilot waves', asserting that every material particle has a wave associated with it, with the frequency and reciprocal wavelength related to the energy and momentum through Planck's constant. Although the assertion was then lacking experimental evidence, Einstein is reported to have said that de Broglie had 'lifted a corner of a great veil'.

In 1924 and 1925 Einstein calculated [10] the fluctuations in a Bose-Einstein gas of material particles, and showed that they exhibited the same structure as the fluctuations in the blackbody spectrum. Einstein saw this as a confirmation of de Broglie's matter waves, and suggested other experimental methods to detect them, one of which was the suggestion of the possibility of the Bose-Einstein condensation.

The formulation of the Bose-Einstein and Fermi-Dirac statistics that govern indistinguishable particles, together with the connection between spin and statistics, provides insights into the statements concerning 'wave-particle duality' that pervade elementary physics textbooks. With a knowledge of quantum statistics, duality is little more than a misleading historical artifact, and discussion of it could be profitably replaced by a simple qualitative discussion of the connection between spin and statistics. This can be done by noting that all fundamental particles possess localized energy and momentum as well as intrinsic periodicities and by considering the following facts.

Entities that possess intrinsic spins that are integer multiples of $\hbar$ have symmetric wave functions and obey Bose-Einstein statistics. This permits ensembles of such entities to exist in a common state with coherent phases, and their members do the same thing at the same time. Thus their macroscopic behavior mimics their microscopic behavior, masking their individualities and revealing their periodic coherences. Early workers used words such as 'fields' and 'waves' to describe what we now call 'bosons'.

Entities that possess intrinsic spins that are half-odd-integer multiples of $\hbar$ have antisymmetric wave functions and obey Fermi-Dirac statistics. This precludes ensembles of such particles from doing the same thing at the same time. Thus they have incoherent phases and their macroscopic behavior differentiates their individualities and averages out their periodicities. Early workers used words such as 'particles' to describe what we now call 'fermions'.

The examples cited in elementary textbooks as illustrative of one or the other of these 'duality' aspects (ocean waves, sound waves, billiard balls, human beings, etc) are themselves constructed granularly from atoms, but simultaneously possess (both individually and collectively) basic periodic frequency modes. Thus the dichotomy of duality may be more a conceptual impediment than a pedagogical tool, since it introduces a counter-experiential distinction for the express purpose of its subsequent refutation.
Another useful conceptual model provided by quantum statistics involves the 'dressed electron'. Electrons and positrons possess spin $\frac{1}{2}$ and are thus fermions, obeying Fermi-Dirac statistics. However, quantum electrodynamics accounts for the electrical interactions among these fermions through the exchange of virtual photons, which are the spin 1 'gauge bosons' that mediate the interaction. Thus there is an inseparable relationship between the electrons and the photons - an electron with no virtual photon accompaniment would have no charge, and would behave like a neutrino. When the electron (which obeys Fermi-Dirac statistics) is taken together with its absorbed and emitted virtual photons (which obey Bose-Einstein statistics), the two together obey Maxwell-Boltzmann statistics.

A simple analogy can be obtained by considering coin flips. If two coins are flipped, each can result in a heads (H) or tails (T) with equal likelihood. Thus the possible outcomes are

$$
\text { HH HT TH TT }
$$

and there is a $25 \%$ chance of either two heads or two tails, and a $50 \%$ chance of one heads and one tails. However, if these are indistinguishable 'quantum coins' (i.e., they have no pre-existence, and only come into being when the wave function collapses in the measurement process), then it is not possible to discriminate between HT and TH. In this case the possible outcomes are

$$
\mathrm{HH} \quad(\mathrm{HT}+\mathrm{TH}) / \sqrt{2} \quad \mathrm{TT}
$$

and there is a $33 \%$ chance of any of the three outcomes of two heads, two tails, or one heads and one tails. This symmetric case is an analogue of the Bose-Einstein distribution. If there is an exclusion principle that precludes both coins from having the same heads or tails property, then the only possible outcome for this simple case is

$$
(\mathrm{HT}-\mathrm{TH}) / \sqrt{2},
$$

which is an antisymmetric analog to Fermi-Dirac statistics. If the Bose-Einstein and Fermi-Dirac distributions are incoherently quantum mechanically averaged (squaring the component of each distribution first and then adding), it yields the same result as the Maxwell-Boltzmann distribution. Similar examples can be constructed with multisided coins (like dice), with the same property that the corresponding average of the Bose-Einstein and the Fermi-Dirac cases yields a Maxwell-Boltzmann analogue.

This quantum coin example has a counterpart in atomic physics in the specification of the triplet and singlet states of a two-valence-electron atom. The spin-triplet obeys Bose-Einstein statistics and the spin-singlet obeys Fermi-Dirac statistics. Since the total wave function of the atom must be antisymmetric under interchange of electron labels, the spatial portion of the wave function must obey the opposite statistics to that of the spin portion. Thus the spatial behavior of the triplet is that of a fermion and the spatial behavior of the singlet is that of a boson. This leads to a tendency for electrons in the triplet to be more spatially separated than those of the singlet; hence the triplet is usually more tightly bound than the singlet. 
It can be a useful conceptual insight into the nature of our physical reality to note that the Maxwell-Boltzmann macroscopic statistical behavior that we observe in daily life subdivides on a microscopic level into Bose-Einstein and Fermi-Dirac components.

\section{Conclusion}

This paper has attempted to place the contributions of Albert Einstein to atomic physics in perspective by considering both the historical context at the time of his work and the developments that have occurred since that time. One thread that is interwoven through the papers that have been considered is the myriad of properties of the photon. As Einstein said, 'For the rest of my life I want to reflect on what light is.'

A similar treatise could be written on almost any field of current physics research. However, the impact of Albert Einstein goes far beyond physics. He captured the popular imagination and was truly the scientist of the people. My father was born in 1905, just a few days after the publication of the fourth paper of the annus mirabilis. Although a pharmacist by profession, he was fascinated by the work of Einstein, and passed that sense of curiosity and wonder on to me.

\section{References}

[1] Einstein A 1905 Uber einen die Erzeugung und Verwandlung des Lichtes betreffenden heuristishen Gesichtspunkt Ann. Phys. 17 132-48

[2] Einstein A 1905 Die von der molekularkinetischen Theorie der Wärme geforderte Bewegung von in ruhenden Flüssigkeiten suspendierten Teilchen Ann. Phys. 17 549-60

[3] Einstein A 1905 Zur Elektrodynamic bewegter Körper Ann. Phys. 17 891-921

[4] Einstein A 1906 Eine neue bestimmung der Molekülardimensionen Ann. Phys. 19 229-47

[5] Einstein A 1909 Zum gegenwärten Stand des Strahlungsproblems Phys. Z. 10 185-93
[6] Einstein A 1909 Uber die Entwicklung unserer Anschauungen über das Weses und die Konsitution der Strahlung Phys. Z. $10817-25$

[7] Einstein A 1916 Die Grundlage der allgemeinen Relativitätstheorie Ann. Phys. 49 769-822

[8] Einstein A 1917 Zur Quantentheorie der Strahlung Phys. Z. 18 121-8

[9] Einstein A 1917 Zum Quantensatz von Sommerfeld und Epstein Verh. Dtsch. Phys. Ges. 19 82-92

[10] Einstein A 1924 Quantentheorie des einatomigen idealen Gases Sitz. Ber. Preuss. Akad. Wiss. (Berlin) 261-7

Einstein A 1925 Sitz. Ber. Preuss. Akad. Wiss. (Berlin) 3-14, $18-25$

[11] Gillispie C G 1997 Pierre-Simon Laplace 1749-1827: A Life in the Exact Sciences (Princeton, NJ: Princeton University Press)

[12] Rigden J S 2005 Einstein 1905: the Standard of Greatness (Cambridge, MA: Harvard University Press)

[13] Lewis G N 1926 The conservation of photons Nature 118 874-5

[14] Shankland R S 1963 Conversations with Albert Einstein Am. J. Phys. 31 47-57

[15] Purcell E M 1963 Electricity and Magnetism, Berkeley Physics Course vol 2 (New York: McGraw-Hill)

[16] Curtis L J 2003 Atomic Structure and Lifetimes: a Conceptual Approach (Cambridge: Cambridge University Press)

[17] Stone A D 2005 Einstein's unknown insight and the problem of quantizing chaos Phys. Today

[18] Ernst A and Hsu J-P 2001 First proposal of the universal speed of light by Voigt in 1887 Chin. J. Phys. 39 211-30

[19] Goldstein H 1980 Classical Mechanics (Reading, MA: Addison-Wesley)

[20] Foldy L L and Wouthuysen S A 1950 On the Dirac theory of spin $\frac{1}{2}$ particles and its non-relativistic limit Phys. Rev. 78 $29-36$

[21] Dirac P A M 1963 The evolution of the physicist's picture of nature Sci. Am. 208 45-54

[22] Feynman R P 1965 The development of the space-time view of quantum electrodynamics Nobel Lecture, 11 December 1965 Online at http://nobelprize.org/physics/laureates/1965/ feynman-lecture.html

[23] Bose S N 1924 Plancks Gesetz und Lichtquantenhypothese Z. Phys. 26178

Bose S N 1924 Z. Phys. 27384

[24] Kleppner D 2005 Rereading Einstein on radiation Phys. Today 5830 